\title{
Spatial photon correlations in multiple scattering media
}

\author{
Smolka, Stephan; Muskens, O.; Lagendijk, A.; Lodahl, Peter
}

Published in:

2010 Conference on Lasers and Electro-Optics (CLEO) and Quantum Electronics and Laser Science Conference (QELS)

Publication date:

2010

Document Version

Publisher's PDF, also known as Version of record

Link back to DTU Orbit

Citation (APA):

Smolka, S., Muskens, O., Lagendijk, A., \& Lodahl, P. (2010). Spatial photon correlations in multiple scattering media. In 2010 Conference on Lasers and Electro-Optics (CLEO) and Quantum Electronics and Laser Science Conference (QELS) (pp. 1-2). IEEE.

\section{General rights}

Copyright and moral rights for the publications made accessible in the public portal are retained by the authors and/or other copyright owners and it is a condition of accessing publications that users recognise and abide by the legal requirements associated with these rights.

- Users may download and print one copy of any publication from the public portal for the purpose of private study or research.

- You may not further distribute the material or use it for any profit-making activity or commercial gain

- You may freely distribute the URL identifying the publication in the public portal

If you believe that this document breaches copyright please contact us providing details, and we will remove access to the work immediately and investigate your claim. 


\title{
Spatial Photon Correlations in Multiple Scattering Media
}

\author{
Stephan Smolka ${ }^{1}$, Otto Muskens ${ }^{2}$, Ad Lagendijk ${ }^{3}$, Peter Lodahl ${ }^{1}$ \\ 1. DTU Fotonik, Department of Photonics Engineering, Technical University of Denmark, \\ Building 345V, 2800 Kgs. Lyngby, Denmark \\ 2. School of Physics \& Astronomy, University of Southampton, Southampton, SO17 1BJ, UK \\ 3. Center for Nanophotonics, FOM-Institute AMOLF, Sciencepark 113, 1098XG Amsterdam, \\ The Netherlands \\ Authore-mail address:stsm@fotonik.dtu.dk
}

\begin{abstract}
We present the first angle-resolved measurements of spatial photon correlations that are induced by multiple scattering of light. The correlation relates multiple scattered photons at different spatial positions and depends on incident photon fluctuations.
\end{abstract}

(C) 2008 Optical Society of America

OCIS codes: (290.4210) Multiple scattering; (030.5290) Photon statistics

\section{Introduction}

Multiple wave scattering is a very interdisciplinary field of research ranging from photonics over acoustics to seismology. Multiply scattered light waves, of concern here, are totally randomized in their direction when they propagate through disordered media like clouds or nanophotonic devices. The waves within the medium interfere with each other, and the light exiting the sample forms a volume speckle pattern. After ensemble averaging over different realizations of disorder, the interference effects tend to vanish, and the light in different directions is uncorrelated. In this case the light transport is described by diffusion theory [1]. Lately, the interest in the quantum nature of multiple scattered light has increased rapidly [2, 3, 4]. Only very recently, spatial photon correlations of genuine quantum origin were observed by multiple scattering of squeezed light based on total transmission quantum noise measurements [5]. Here we present a detailed investigation of the first direct measurement of spatial photon correlations induced by multiple scattered light.

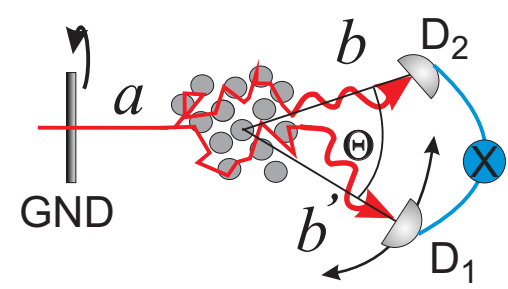

Fig. 1. Sketch of the experimental setup to measure spatial photon correlations in multiple scattering media. GND: grinded glass plate; D: detector consisting of adjustable iris, polarizer, $10 \mathrm{~nm}$ interference filter, and single photon detector; $a$ : incident light channel; $b, b^{\prime}:$ exit light channels. $\Theta$ defines the relative angle between the directions of multiply scattered light that is recorded with the detectors.

The spatial photon correlation function between different directions $b, b^{\prime}$ (see Fig 1 ) is defined as

$$
\overline{C_{a b a b^{\prime}}^{Q}}=\frac{\overline{\left\langle\hat{n}_{a b} \hat{n}_{a b^{\prime}}\right\rangle}}{\overline{\left\langle\hat{n}_{a b}\right\rangle} \times \overline{\left\langle\hat{n}_{a b}\right\rangle}},
$$

where $a$ labels the direction of the incident light. The operator $\hat{n}_{a b}$ represents the number of photons in output mode $b$, and $\langle\ldots\rangle$ denotes the quantum mechanical expectation value while the bar refers to an average over all realizations of disorder. Using a full quantum mechanical model of multiple scattering of light [4], the spatial photon correlation function can be expressed by

$$
\overline{C_{a b a b^{\prime}}^{Q}}=\left[\frac{\Delta n_{a}^{2}}{\left\langle\hat{n}_{a}\right\rangle^{2}}+1\right] \times \overline{C_{a b a b^{\prime}}} .
$$

Here $\hat{n}_{a}$ represents the number of incident photons and $\Delta n_{a}^{2}$ is the variance of the incident photon fluctuations. $\overline{C_{a b a b^{\prime}}}$ are classical intensity correlations between direction $b$ and $b^{\prime}$ that are induced by the multiple scattering medium [1]. 


\section{QMG3.pdf}

\section{Experiment}
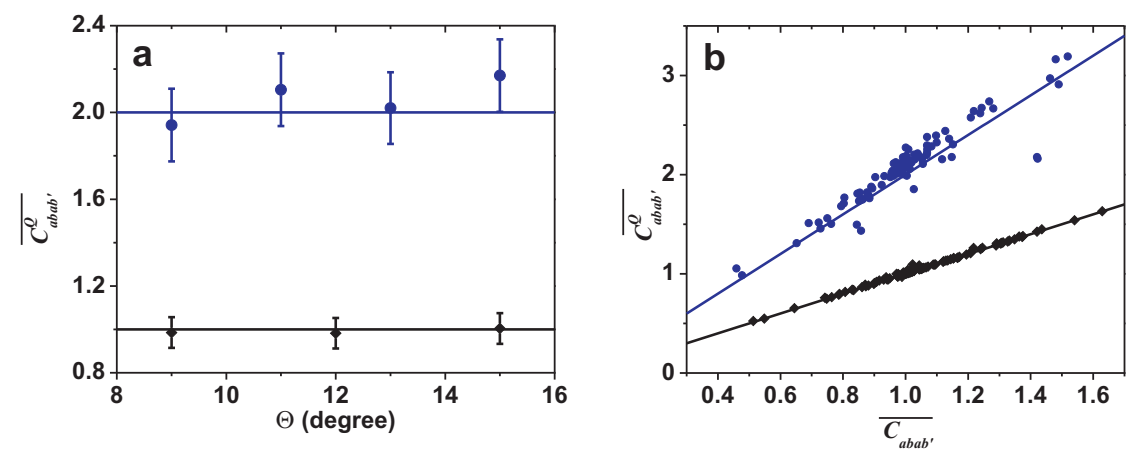

Fig. 2. a, Angle dependence of the spatial photon correlation function $\overline{C_{a b a b^{\prime}}^{Q}}$ for a coherent light source (black squares) and a Gaussian light source (blue circles) after ensemble averaging over $N=200$ speckle patterns. Detector $\mathrm{D}_{1}$ is rotated behind the sample to change $\Theta$. b, $\overline{C_{a b a b^{\prime}}^{Q}}$ as a function of the spatial intensity correlation function $\overline{C_{a b a b^{\prime}}}$ for a coherent light source (black squares) and a Gaussian radiation source (blue circles) after averaging over two speckle patterns. The predicted theory in a,b is given by straight lines without any free parameters assumed.

A Ti-Sapphire laser $(\lambda=780 \mathrm{~nm})$ serves as a light source with Poissonian photon statistics $\left(\Delta n_{a}^{2}=\left\langle n_{a}\right\rangle\right)$. We can generate, e.g., a Gaussian light source obeying $\Delta n_{a}^{2}=\left\langle n_{a}\right\rangle+\left\langle n_{a}\right\rangle^{2}$, by positioning a slowly rotating grinded glass plate in the beam path (Fig. 1). The rotating grinded glass plate generates a superposition of coherent beams with random amplitudes and phases resulting in Super-Poissonian photon statistics. The light beam is focussed on the front surface of a multiple scattering medium which consists of $\mathrm{TiO}_{2}$ nano-particles (sample length $L=(6.3 \pm 0.2) \mu \mathrm{m}$, average distance between two scattering events $\ell=(0.9 \pm 0.1) \mu \mathrm{m})$. The multiply scattered light of different directions is recorded with two single photon detectors which are connected to a photon counting module to calculate $\left\langle\hat{n}_{a b} \hat{n}_{a b^{\prime}}\right\rangle$ and $\left\langle\hat{n}_{a b}\right\rangle$, see Eq. (1). The ensemble average is obtained by probing different sample positions. Since $L \gg \ell$, the light intensity transport can be described by diffusion theory and classical intensity correlations vanish, hence $\overline{C_{a b a b^{\prime}}}=1$.

Figure 2a displays the measured photon correlations for a coherent and a Gaussian light source as function of the relative angle $\Theta$ between both detectors. We observe that $\overline{C_{a b a b^{\prime}}^{Q}}$ does not depend on $\Theta$. The data are in excellent agreement with theory which predicts that the correlation function is infinite in range. To prove the dependency of $\overline{C_{a b a b^{\prime}}^{Q}}$ on spatial intensity correlations we measure the fluctuations in the spatial photon correlation function, caused by a finite number of ensemble averages, $N$. We record many different measurements, each measurement consisting of an ensemble average over only two realizations of disorder. Simultaneously we determine the classical intensity correlation function for these measurements from the sample transmission coefficients $T_{a b}$ in direction $b$ and $b^{\prime}$ [1]. The results are plotted in Fig. $2 \mathrm{~b}$. We observe a linear dependency of the photon correlation function on $\overline{C_{a b a b^{\prime}}}$ which is in very good agreement to theory as expected from Eq. (2). Note, that spatial intensity correlations between different directions are only present because we used a very limited number of ensemble averages, otherwise they vanish.

\section{Conclusion}

We have presented the direct measurement of spatial photon correlations that are induced by multiply scattered light in different directions. The correlations were found to be infinite in range, dependent on the photon statistics of the incident light source, and dependent on spatial intensity correlations induced by the multiple scattering medium. The experimental results were found to be in excellent agreement with the full quantum optics theory for multiple scattered light.

\section{References}

[1] M.C.W. van Rossum and T.M. Nieuwenhuizen, Rev. Mod. Phys. 71, 313 (1999).

[2] J. Tworzydlo and C.W.J. Beenakker, Phys. Rev. Lett. 89, 043902 (2002).

[3] P. Lodahl and A. Lagendijk, Phys. Rev. Lett. 94, 153905 (2005).

[4] P. Lodahl, A.P. Mosk, and A. Lagendijk, Phys. Rev. Lett. 95, 173901 (2005).

[5] S. Smolka, A. Huck, U.L. Andersen, A. Lagendijk, and P. Lodahl, Phys. Rev. Lett. 102, 193901 (2009). 